\title{
Effects of poly(L-lactide- $\varepsilon$-caprolactone) and magnesium hydroxide additives on physico-mechanical properties and degradation of poly(L-lactic acid)
}

\author{
Eun Young Kang ${ }^{1,2}$, Eugene Lih', Ik Hwan Kim², Yoon Ki Joung ${ }^{1,3}$ and Dong Keun Han ${ }^{1,3^{*}}$
}

\begin{abstract}
Background: Biodegradable poly(L-lactic acid) (PLLA) is one of the most widely used polymer in biomedical devices, but it still has limitations such as inherent brittleness and acidic degradation products. In this work, PLLA blends with poly(L-lactide- $\varepsilon$-caprolactone) $(\mathrm{PLCL})$ and $\mathrm{Mg}(\mathrm{OH})_{2}$ were prepared by the thermal processing to improve their physico-mechanical and thermal properties. In addition, the neutralizing effect of $\mathrm{Mg}(\mathrm{OH})_{2}$ was evaluated by degradation study.

Results: The elongation of PLLA remarkably increased from 3 to $164.4 \%$ and the glass transition temperature $\left(T_{q}\right)$ of PLLA was slightly reduced from 61 to $52{ }^{\circ} \mathrm{C}$ by adding $\mathrm{PLCL}$ additive. $\mathrm{Mg}(\mathrm{OH})_{2}$ in polymeric matrix not only improved the molecular weight reduction and mechanical strength of PLLA, but also neutralized the acidic byproducts generated during polyester degradation.

Conclusions: Therefore, the results demonstrated that the presence of PLCL and $\mathrm{Mg}(\mathrm{OH})_{2}$ additives in PLLA matrix could prevent the thermal decomposition and control degradation behavior of polyester.
\end{abstract}

Keywords: Poly(L-lactic acid), Poly(L-lactide- $\varepsilon$-caprolactone), Magnesium hydroxide, Thermal decomposition, Neutralization

\section{Background}

Poly(L-lactic acid) (PLLA) has excellent mechanical properties, thermal plasticity, and transparency, which is a renewably derived thermoplastic and biodegradable polyester extensively investigated over the last several decades [1-3]. However, a few drawbacks of PLLA including inherent brittleness, poor melt strength, and imperfect biocompatibility have limited numerous applications in biomedical science and engineering such as implant devices, tissue scaffolds, and internal sutures $[4,5]$. In particular, thermal extrusion process at high temperature (Fig. 1a), which is necessary to manufacture various biodegradable devices based on PLLA, maximizes inherent

\footnotetext{
* Correspondence: dkh@kist.re.kr

${ }^{1}$ Center for Biomaterials, Korea Institute of Science and Technology, Hwarangno 14-gil 5, Seongbuk-gu Seoul 02792, South Korea

${ }^{3}$ Department of Biomedical Engineering, Korea University of Science and Technology, Gajeong-ro 217, Yuseong-gu Daejeon 34113, South Korea

Full list of author information is available at the end of the article
}

flaws of PLLA such as poor mechanical properties, matrix cracking, and thermal instability via random chain scission [6], depolymerisation [7] and intramolecular transesterification [7, 8]. Fig. $1 \mathrm{~b}$ and $\mathrm{c}$ show the degradation mechanism of PLLA based on backbiting and hydrolytic degradations. The acidic byproducts generated in this reaction acted as a nucleophile in ester linkage and accelerated the degradation reaction of PLLA backbone. Moreover, the presence of moisture in PLLA accelerates the cleavage of the ester linkage with the acid and alcohol groups that induced a significant molecular weight reduction. In biomedical applications, the released acidic degradation products from PLLA matrix reduce the $\mathrm{pH}$ of implant environment and result in inflammatory reaction in the body [9].

One of the most important disadvantages of PLLA is brittleness due to its semi-crystalline character, which can limit its applicability. Many papers have reported 
a

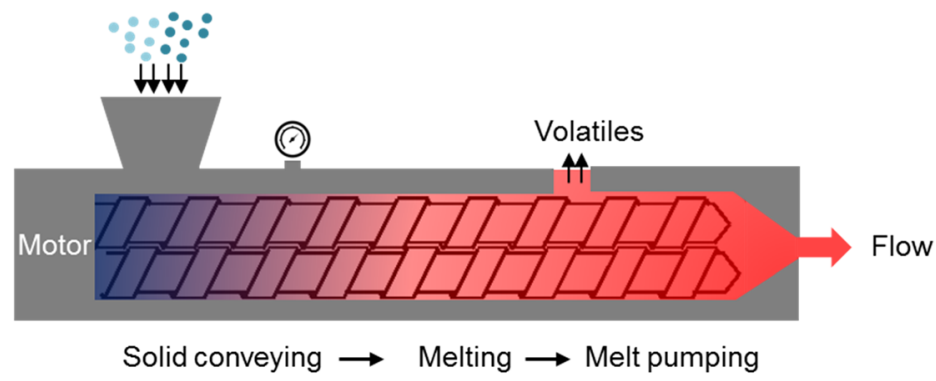

b

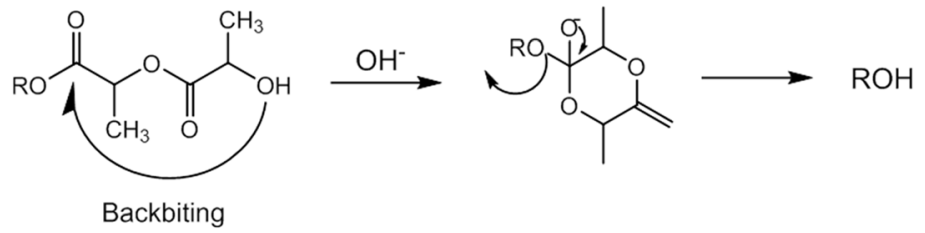

C

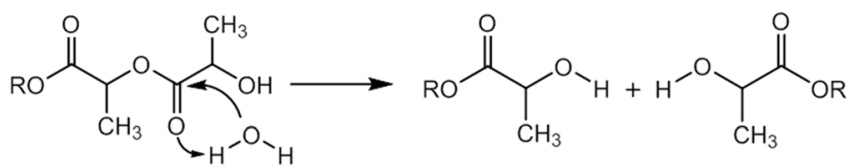

Thermal hydrolysis

Fig. 1 a Thermal extrusion process and $\mathbf{b}, \mathbf{c}$ degradation mechanisms of PLLA

the PLLA blends with ductile and biodegradable polymers such as polyglycolic acid (PGA) [10, 11], polyamide 6 (PA6) [12] and poly(butylene succinate-co-adipate) (PBSA) [13, 14]. Aliphatic polyester based on polycaprolactone such as poly(L-lactide- $\varepsilon$-caprolactone) (PLCL) has been used to improve the processability and thermal stability of PLLA because PLCL has flexible characteristics, low melting temperature, and aliphatic chain of polycaprolactone segment. The PLLA blends with polycaprolactone based polyester exhibit remarkable increase in their elongation at break due to relative increase of the amorphous region compared with PLLA [15-17].

Magnesium hydroxide, which is widely used as an antacid agent, is a basic inorganic compound with the chemical formula of hydrated $\mathrm{Mg}(\mathrm{OH})_{2} \cdot \mathrm{Mg}(\mathrm{OH})_{2}$ is in vivo absorbable in the form of magnesium and hydroxide ions as well as biocompatible [18]. The magnesium ions can be interact with anionic compounds and neutralize the acidic degradation products of PLLA [19]. In addition, $\mathrm{Mg}(\mathrm{OH})_{2}$ also can be used to mechanically reinforce the PLLA matrix, which is a key factor resulting in effective improvement in mechanical properties [20].

In this work, the PLLA blends with PLCL and $\mathrm{Mg}(\mathrm{OH})_{2}$ additives were developed by thermal processing to improve the flexibility and thermal stability of PLLA matrix and enhance the tensile strength of thermally-processed PLLA. The physic-mechanical properties of PLLA control and PLLA/PLCL blends were measured by gel permeation chromatography (GPC) and universal testing machine (UTM), and their thermal properties were evaluated by using differential scanning calorimetry (DSC) and thermogravimetric analysis (TGA). The effect of $\mathrm{Mg}(\mathrm{OH})_{2}$ in PLLA and PLLA/ PLCL matrix was determined by measuring molecular weight and mechanical properties such as tensile strength and elongation. The degradation study was performed, and the degraded samples were assessed by following $\mathrm{pH}$ change and calculating molecular weight reduction to evaluate the the neutralizing effect of $\mathrm{Mg}(\mathrm{OH})_{2}$.

\section{Methods \\ Materials}

Poly(L-lactide) raw material (PLLA, average $\mathrm{Mw}=380$ $\mathrm{kDa}$ ) was supplied by Samyang Co. (Seoul, Korea). Poly(L-lactide-co- $\varepsilon$-caprolactone) (PLCL, average $\mathrm{Mw}=$ $380 \mathrm{kDa}$ ) composed with 50:50 or 75:25 ratio of Llactide and $\varepsilon$-caprolactone units was obtained from DURECT Co. (Birmingham, AL, USA). Chloroform and magnesium hydroxide $\left(\mathrm{Mg}(\mathrm{OH})_{2}\right)$ were purchased by Baker Chemicals Inc. (Center Valley, PA, USA) and Junsei Chemical Co., Ltd. (Tokyo, Japan), respectively. All chemicals were reagent grade and used without further purification.

\section{Fabrication of PLLA/PLCL/Mg(OH $)_{2}$ blends}

The PLLA, PLCL, and $\mathrm{Mg}(\mathrm{OH})_{2}$ were mixed by solvent casting method to form a homogenous mixture. First, PLLA pellets were dried in a vacuum oven at $60{ }^{\circ} \mathrm{C}$ for $24 \mathrm{~h}$ to prevent hydrolysis caused by residual moisture 
Table 1 Various blend compositions of PLCL and $\mathrm{Mg}(\mathrm{OH})_{2}$ in PLLA

\begin{tabular}{lllll}
\hline Abbreviation & PLLA(wt\%) & PLCL (50:50)(wt\%) & PLCL (75:25)(wt\%) & Mg(OH) $2(w t \%)$ \\
\hline Raw PLLA & 100 & - & - & - \\
Processed PLLA & 100 & - & - & - \\
PLLA95/PLCL5 (50:50) & 95 & 5 & - & - \\
PLLA90/PLCL10 (50:50) & 90 & 10 & - & - \\
PLLA85/PLCL15 (50:50) & 85 & 15 & - & - \\
PLLA80/PLCL20 (50:50) & 80 & 20 & 5 & - \\
PLLA95/PLCL5 (75:25) & 95 & - & 10 & - \\
PLLA90/PLCL10 (75:25) & 90 & - & 15 & - \\
PLLA85/PLCL15 (75:25) & 85 & - & 20 & - \\
PLLA80/PLCL20 (75:25) & 80 & - & 10 & 5 \\
PLLA90/PLCL10/Mg5 & 90 & - & \\
\hline
\end{tabular}

and acidic monomer. Dried PLLA raw materials and PLCL (50:50 or $75: 25$, respectively) additives were dissolved in $80 \mathrm{~mL}$ of chloroform with different rations of PLLA and PLCL, which the total amount of solutes was $5 \mathrm{~g}$ in the solution. For $\mathrm{Mg}(\mathrm{OH})_{2}$ incorporated samples, $0.25 \mathrm{~g}$ of $\mathrm{Mg}(\mathrm{OH})_{2}$ powders were added into the above polymer solutions. The mixture was poured into the Teflon mold $\left(100 \times 90 \times 15 \mathrm{~mm}^{3}\right)$, and then the solvent was slowly evaporated at room temperature for $24 \mathrm{~h}$. The film samples were dried under vacuum at $60{ }^{\circ} \mathrm{C}$ to remove the residual solvent. The homogeneously mixed composites based on PLLA were chopped up into small fragments under $5 \mathrm{~mm}$, and then kept at room temperature in vacuo until thermal process. Pure PLLA fragments were also prepared using the same method. Table 1 lists the abbreviation and conditions of samples including raw PLLA, processed PLLA, PLLA/PLCL, PLLA $/ \mathrm{Mg}(\mathrm{OH})_{2}$, and PLLA/PLCL/Mg(OH $)_{2}$ blends.

The PLLA only, PLLA/PLCL, PLLA/Mg(OH $)_{2}$, and PLLA/PLCL/Mg $(\mathrm{OH})_{2}$ composites underwent the thermally melting and hot-pressing process using Carver Press 30-12H (Carver Inc., Wabash, IN, USA). Four grams of polymer mixture put on the hot-press plate, and the plate temperature was then raised to $190{ }^{\circ} \mathrm{C}$. After melting time of $3 \mathrm{~min}$, the pressure increased to about 7 tons and maintained for press time of $6 \mathrm{~min}$. The dimension of compressively molded specimens was $150 \times 150 \mathrm{~mm}^{2}$ and its thickness was $200 \mu \mathrm{m}$. All specimens were stored in vacuum oven.

\section{Characterizations of PLLA/PLCL/Mg(OH $)_{2}$ blends}

To evaluate the effects of PLCL and $\mathrm{Mg}(\mathrm{OH})_{2}$ additives on the prevention of the molecular weight $(\mathrm{Mw})$ of PLLA during thermal processing, $\mathrm{Mw}$ of processed PLLA and PLLA blends was measured by gel permeation chromatography (GPC). The sample was dissolved in chloroform (HPLC grade) at $3 \mathrm{mg} / \mathrm{mL}$ concentration, and then the polymer solutions were filtered with a 0.45 $\mu \mathrm{m}$ PTFE filter to remove dust and contaminants. The GPC measurements were performed on an equipment consisted of a Waters 515 HPLC pump, a Waters 717 plus auto sampler and a Waters 410 refractive index (RI) detector (Waters, USA) with KF $804 \mathrm{~L}$ and KF 803 (7 $\mu \mathrm{m}$, $8 \times 300 \mathrm{~mm}$, Shodex, Japan) columns (molecular weight resolving range of 500-400,000 Da). The GPC columns were performed using chloroform as the eluent with flow rate of $1 \mathrm{~mL} / \mathrm{min}$, and $\mathrm{Mw}$ were calibrated with polystyrene standard.

The mechanical properties such as tensile strength and elongation were measured by a universal testing machine (UTM, Instron Co., USA) in accordance with ASTM standard D638. For dynamic tensile test, the processed PLLA and composites with PLLA and additives were prepared with dumbbell shaped specimens (45 $\times 6 \times 2 \mathrm{~mm}^{3}$, length $\times$ width $\times$ thickness) cut by a punch of a compression-molding machine. All test samples were tested after 1 week to remove the internal stress. The specimens were carried out with crosshead speed of $4 \mathrm{~mm} / \mathrm{min}$ till failure to determine the influence of additives in PLLA on mechanical property.

The thermal properties of processed PLLA and PLLA blends were investigated by differential scanning calorimetry (DSC, TA Instrument, USA) and thermogravimetric analyser (TGA, TA Instruments, USA), respectively. The glass transition temperature $\left(T_{\mathrm{g}}\right)$ of samples was determined by using DSC analysis. The temperature scan was performed with a heating and cooling rate of $10{ }^{\circ} \mathrm{C} / \mathrm{min}$ under nitrogen atmosphere. The samples were heated from 30 to $210{ }^{\circ} \mathrm{C}$, held for 1 min to erase thermal history effects and cooled to $30^{\circ} \mathrm{C}$, held for $1 \mathrm{~min}$ and then heated to $210{ }^{\circ} \mathrm{C}$ again for the second scan, which was used to determine the $T_{g}$. The effects of additives types and contents on the thermal stability were assessed through TGA. The melting $\left(\mathrm{T}_{\mathrm{m}}\right)$ and crystallization $\left(\mathrm{T}_{\mathrm{c}}\right)$ temperatures, in addition to the associated enthalpy of melting $\left(\Delta \mathrm{H}_{\mathrm{m}}\right)$ were measured 


\section{a}

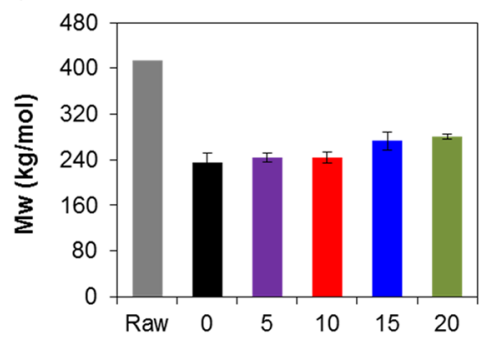

PLCL(50:50) contents (wt.\%)

C

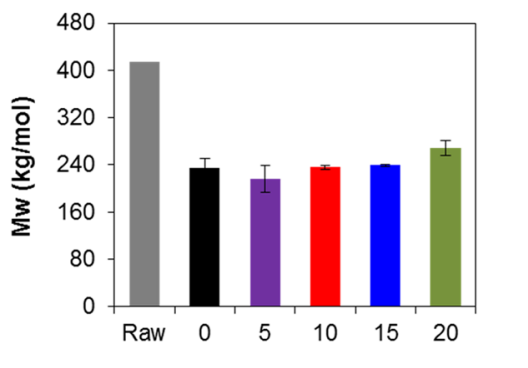

b

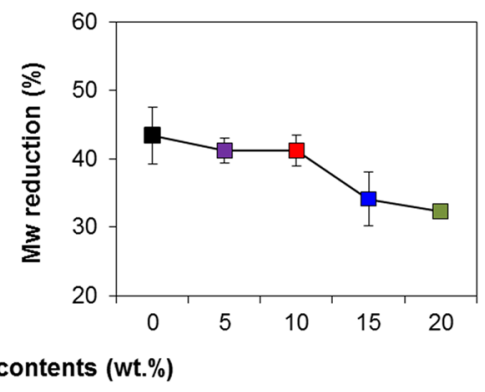

d

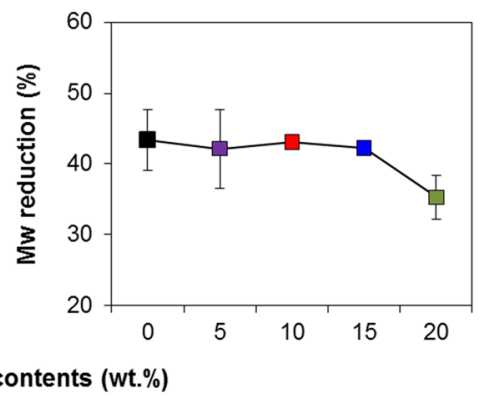

Fig. 2 Molecular weight properties of PLLA blends with various a, b PLCL (50:50) and c, d PLCL (75:25) contents

using a heating/cooling ramp from 20 to $600{ }^{\circ} \mathrm{C}$. A heating rate of $10{ }^{\circ} \mathrm{C} / \mathrm{min}$ was used under nitrogen atmosphere and at a flow rate of $20 \mathrm{~mL} / \mathrm{min}$. Dry sample weighing about $1 \mathrm{mg}$ was used. The standard uncertainty of the sample mass measurement is $\pm 1 \%$.

\section{Degradation test}

To study the degradation behavior including $\mathrm{pH}$ change, mass loss, and molecular weight reduction of PLLA and PLLA blends, four groups (PLLA, PLLA/PLCL, PLLA/ $\mathrm{Mg}(\mathrm{OH})_{2}$, PLLA/PLCL/Mg(OH $\left.)_{2}\right)$ were prepared in replicate experiments $(n=20$, each). Pre-weighted specimens $\left(W_{O}\right)$ were immersed into $5 \mathrm{~mL}$ of phosphatebuffered saline (PBS, pH 7.4) solution at $60{ }^{\circ} \mathrm{C}$ under accelerated weathering conditions $[21,22]$. The $\mathrm{pH}$ of the PBS solution was monitored every 2 days by a $\mathrm{pH}$ meter (Hanna Instrument, USA). Temperature and $\mathrm{pH}$ of the PBS solution were monitored during the degradation period and they remained at $37^{\circ} \mathrm{C}$ and 7.4 , respectively.

The $\mathrm{pH}$ change, was measured at the predetermined time point $(1,3,5$, and 7 days) to confirm acidity of samples as well as the neutralization of lactic acids with $\mathrm{Mg}(\mathrm{OH})_{2}$. At time intervals, the specimens were accurately weighed after deionized water rinse and vacuum drying for $48 \mathrm{~h}\left(W_{t}\right)$ to determine the mass loss and the reduction of molecular weight. The residual weight percentage was calculated according to the following equation: Mass loss $(\%)=\left(W_{O}-W_{t}\right) / W_{O} \times 100$ [23]. The reduced molecular weight of degraded specimens $\left(M_{t}\right)$ was estimated using GPC and compared with an initial molecular weight of samples $\left(M_{0}\right)$. The reduction of molecular weight (\%) was also calculated according to the following equation: $\mathrm{Mw}$ reduction (\%) = $\left(M_{O}-M_{t}\right) / M_{O} \times 100$.

\section{Statistical analysis}

The data were presented as mean \pm standard deviation (SD). The results obtained by ANOVA were carried out using origin programs. The significance level considered was 0.05 and data groups were different from others.

\section{Results and Discussion PLLA/PLCL blends}

The molecular weight and reduction degree of thermally processed PLLA and PLLA/PLCL blends with different feed ratios were shown in Fig. 2. The molecular weight of processed PLLA rapidly decreased to 234,342 $\mathrm{Da}$ as compared with raw PLLA $(414,256 \mathrm{Da})$, which is mainly caused by random scission to generate acidic oligomers and monomers under thermal processing [6]. These oligomers and monomers with carboxyl and hydroxyl end groups as nucleophiles could attack ester linkages on PLLA backbone via intermolecular transesterification and backbiting, and the decomposition of PLLA matrix was accelerated by increasing acidic byproducts and esterification reaction [24]. In contrast, the addition of PLCL (50:50 or 75:25) with increasing PLCL content (5$20 \mathrm{wt} \%)$ into PLLA matrix slightly increased the molecular weight of PLLA blends after thermal processing compared 
with processed PLLA (Fig. 2a and c). With reflecting these results, the reduction degree of molecular weight gradually decreased with increase in PLCL ratio of PLLA/ PLCL blends (Fig. 2b and d). Especially, PLCL (50:50) was more effective than PLCL (75:25) on inhibition of molecular weight reduction. The increased proportion of $\varepsilon$-caprolactone in polymer blends tended to reduce the thermal degradation due to greater thermal stability of $\varepsilon$-caprolactone than that of lactic acid [25]. That is why aliphatic polycaprolactone block in PLCL has one ester linkage with hexanoate repeat unit, whereas polyester PLLA has two ester linkages within repeat unit. This result supposed that the thermal decomposition and formation of acidic byproducts of PLLA/PLCL blends occurred much less than only PLLA at high temperature, and the molecular weight reduction of PLLA/PLCL blends was consequently prevented.

The mechanical properties such as tensile strength and elongation of PLLA/PLCL blends with different PLCL contents were evaluated as compared with thermally processed PLLA as a control. Figure 3 showed that the processed PLLA has higher tensile strength than PLLA/PLCL blends, while the elongation at break of the processed PLLA is severely low compared with PLLA/ PLCL blends. It also is observed that the tensile strength decreased, whereas elongation dramatically increased with increasing proportion of PLCL (50:50 or 75:25) in PLLA/PLCL blends. The PLLA exhibited tensile strength of 54.4 MPa. The tensile strength decreased to 24.6 and
44.2 MPa by blending $20 \mathrm{wt} \%$ of PLCL (50:50) and PLCL (75:25) in PLLA, respectively (Fig. $3 \mathrm{a}$ and c). The elongation of PLLA/PLCL (50:50) blends with different ratios in 95:5, 90:10, 85:15, and 80:20 was 4.8, 31.1, 158.7, and $160.4 \%$, respectively (Fig. 3b). In PLLA/PLCL (75:25) blends, the elongation increased from 6.4 to $48.8 \%$ with increasing PLCL contents from 5 to $20 \%$, compared with PLLA (3.0 \%) (Fig. 3d). The result demonstrated that PLCL as a plasticizer could improve the flexibility of PLLA but remarkably reduce the tensile strength. In particular, the tensile strength of PLLA/PLCL (50:50) was thoroughly reduced, which could be limited to the application being considered. On the other hands, PLCL (75:25) blended PLLA relatively exhibited similar tensile strength to only PLLA with high flexibility. It is well known that polycaprolactone is highly amorphous, imparting flexibility comparable to a semi-crystalline PLLA. In PLLA and PLCL blended matrix, the matrix became flexible with increasing proportion of polycaprolactone. The ratio between semi-crystalline PLLA and amorphous polycaprolactone is important for proper mechanical property, because the amorphous structure makes the matrix not only flexible but also weak. PLCL (50:50) is composed of twice more caprolactone content than that of PLCL (75:25). Therefore, the tensile strength of PLLA blending with PLCL (50:50) was lower than that of PLLA blending with PLCL (75:25). In mechanical properties results, the PLLA90/PLCL10 (50:50 and $75: 25)$ exhibited to successfully prevent the molecular

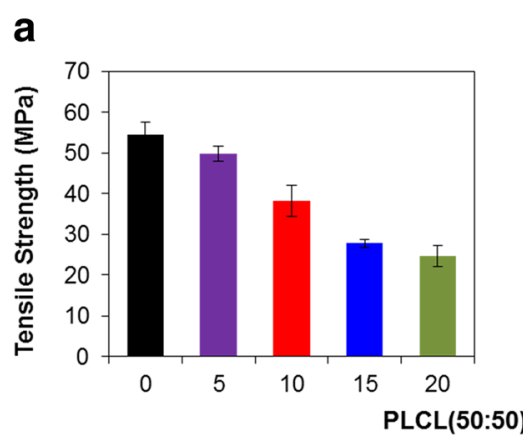

b

C

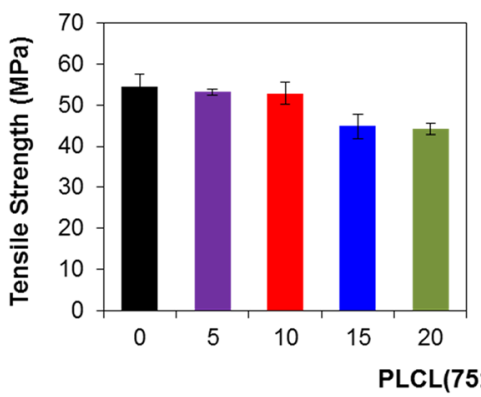

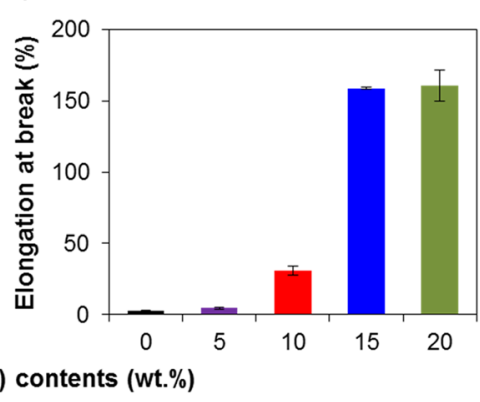

d

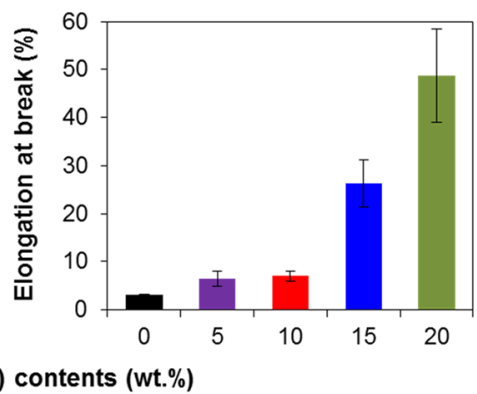

Fig. 3 Mechanical properties of PLLA blends with various a, b PLCL (50:50) and c, d PLCL (75:25) contents 
Table 2 Thermal properties of PLLA control and PLLA/PLCL blends

\begin{tabular}{llll}
\hline Material & $\mathrm{T}_{\mathrm{g}}\left({ }^{\circ} \mathrm{C}\right)$ & $\mathrm{T}_{\mathrm{m}}\left({ }^{\circ} \mathrm{C}\right)$ & $\Delta \mathrm{H}_{\mathrm{m}}(\mathrm{J} / \mathrm{g})$ \\
\hline Raw PLLA & 59 & 176 & 49 \\
Processed PLLA & 61 & 178 & 52 \\
PLLA95/PLCL5 (50:50) & 58 & 177 & 41 \\
PLLA90/PLCL10 (50:50) & 56 & 177 & 36 \\
PLLA85/PLCL15 (50:50) & 55 & 175 & 32 \\
PLLA80/PLCL20 (50:50) & 52 & 174 & 28 \\
PLLA95/PLCL5 (75:25) & 58 & 177 & 54 \\
PLLA90/PLCL10 (75:25) & 57 & 177 & 46 \\
PLLA85/PLCL15 (75:25) & 55 & 177 & 38 \\
PLLA80/PLCL20 (75:25) & 55 & 176 & 34 \\
\hline
\end{tabular}

$\mathrm{T}_{\mathrm{g}}$ : glass transition temperature; $\mathrm{T}_{\mathrm{m}}$ : melt temperature; $\Delta \mathrm{H}_{\mathrm{m}}$ : heat of melting

weight reduction and to properly enhance the flexibility of PLLA.

The thermal analysis of PLLA/PLCL blends was identified by DSC and confirmed by TGA. Table 2 summarized the thermal characteristics including the glass transition temperature $\left(\mathrm{T}_{\mathrm{g}}\right)$, melting temperature $\left(\mathrm{T}_{\mathrm{m}}\right)$, and melting enthalpy $\left(\Delta \mathrm{H}_{\mathrm{m}}\right)$ of processed PLLA and PLLA/PLCL blends, acquired from DSC in second heating scan. The results indicated that $\mathrm{T}_{\mathrm{g}}$ and $\Delta \mathrm{H}_{\mathrm{m}}$ of PLLA/PLCL (50:50 or 75:25) blends decreased with increasing PLCL content, an endothermic melting peak indicated around $177{ }^{\circ} \mathrm{C}$. The values of $\mathrm{T}_{\mathrm{g}}$ and $\Delta \mathrm{H}_{\mathrm{m}}$ of PLLA were measured to be $61{ }^{\circ} \mathrm{C}$ and $52 \mathrm{~J} / \mathrm{g}$, respectively. $\mathrm{T}_{\mathrm{g}}$ value not only decreased by adding PLCL but also gradually decreased with increasing PLCL content. Moreover, the value of $\Delta \mathrm{H}_{\mathrm{m}}$ decreased from 52 to 28 and $34 \mathrm{~J} / \mathrm{g}$ for PLCL $(50: 50)$ and PLCL $(75: 25)$ corresponding to PLCL content from 0 to $20 \mathrm{wt} \%$, which clearly indicated the reduced PLLA crystallization with PLCL. Moreover, $T_{m}$ value of PLLA slightly decreased with the addition of PLCL. As described above, the thermal properties such as $T_{g}, \Delta H_{m}$ and $T_{m}$ strongly influenced by polymer molecular structure [26]. In general, decreasing the crystalline of a semi-crystalline polymer is accompanied by decrease in modulus, stiffness, yield stress, melting point, $\mathrm{T}_{\mathrm{g}}$, and dimensional stability, and by increase in impact resistance, elongation, and thermal expansion [27]. These results in semi-crystalline PLLA/ PLCL blends could be attributed to the decreasing crystalline of PLLA with addition of PLCL containing amorphous polycaprolactone black, as shown in Fig. 4. Although the crystalline domain imparted much mechanical strength and stability to the PLLA/PLCL matrix, the amorphous structure absorbs a substantial fraction of the applied strain energy, owing to its higher elastic compliance [28].

TGA was also carried out on the different formulations to determine the effect of PLCL $10 \mathrm{wt} \%$ (50:50 or 75:25) on the thermal stability of PLLA (Fig. 5). It revealed that the decomposition temperature of PLLA90/ PLCL10 blends was slightly high compared to the processed PLLA control. The addition of PLCL (50:50 or $75: 25)$ affected the thermal stability of PLLA at high temperature ranges over $290{ }^{\circ} \mathrm{C}$. The thermal degradation of PLLA90/PLCL10 blends occurred through ester pyrolysis reaction and induced chain cleavages to be randomly distributed $[29,30]$. Since the proton in the $\mathrm{CH}$ group of the main chain of PLLA is labile, it has been suggested that the proximity of this labile proton to the ester group affects the thermal sensitivity of the polymer. Thus, the PLLA blending with PLCL with one ester linkage per one repeating unit was more thermally stable than only PLLA with two ester linkage per one repeating unit. In addition, PLLA90/PLCL10 (50:50) has a relatively high thermal stability as compared with others due to the lower proportion of polylactide in whole matrix. Although flexibility and thermal properties of PLLA blending with PLCL (50:50) were better than that of PLLA/PLCL (75:25), its mechanical strength was severely poor for applying to a polymeric biomaterial in medical applications. In this regard, further study on PLLA/PLCL/Mg $(\mathrm{OH})_{2}$ matrix will be carried out by using PLLA90/PLCL10 blends with PLCL ratio of 75:25 due to its proper mechanical property.

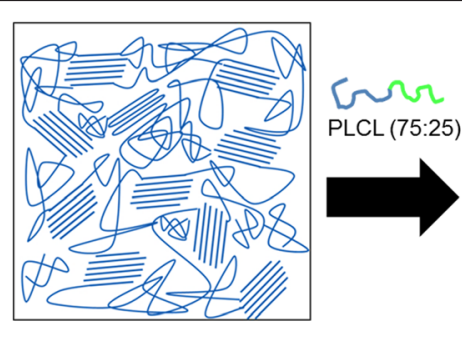

PLLA

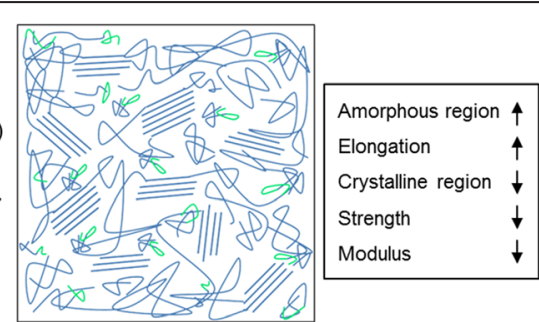

PLLA/PLCL

Fig. 4 Illustration of the preparation of PLLA/PLCL (75:25) composite film with improved processibility 


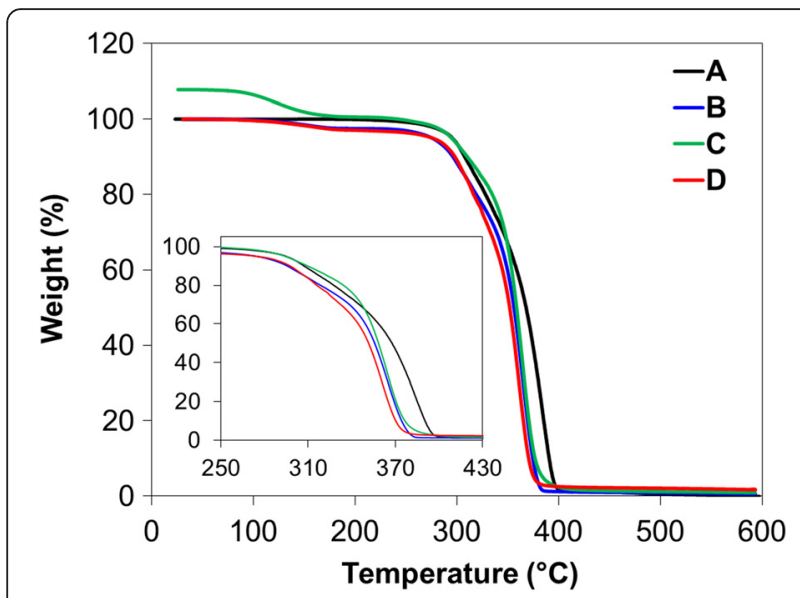

Fig. 5 The TGA thermograms of processed PLLA and PLLA blends: a Raw PLLA, b Processed PLLA, c PLLA90/PLCL10 (50:50), and d PLLA90/PLCL10 (75:25) blends

\section{PLLA/PLCL/Mg(OH) 2 blends}

The PLCL (75:25) and $\mathrm{Mg}(\mathrm{OH})_{2}$ (coded to $\mathrm{Mg}$ ) blended PLLA samples were fabricated by thermal processing. PLLA90/PLCL10/Mg5 composite was characterized and compared with thermally processed PLLA, PLLA90/ PLCL10, and PLLA100/Mg5 samples. Figure 6a and b were observed that the thermal decomposition of PLLA was alleviated by adding $\mathrm{Mg}(\mathrm{OH})_{2}$ as well as PLCL. The molecular weight of PLLA100/Mg5 and PLLA90/ PLCL10/Mg5 increased compared with processed PLLA, and thus the reduction degree greatly decreased, which indicated that $\mathrm{Mg}(\mathrm{OH})_{2}$ improved the thermal stability. $\mathrm{Mg}(\mathrm{OH})_{2}$ as a basic inorganic compound degraded as $\left[\mathrm{Mg}^{2+}\right]$ and $\left[\mathrm{OH}^{-}\right]$. One $\mathrm{Mg}^{2+}$ of $\mathrm{Mg}(\mathrm{OH})_{2}$ also needs two anionic compounds to neutralize the charge and thus binds to anionic terminal group of PLLA and/or acidic degradation product; $\left[\mathrm{Mg}^{2+}\right] \cdot 2\left[\right.$ lactic acid- $\left.\mathrm{OH}^{-}\right]$. The $\left[\mathrm{Mg}^{2+}\right] \cdot 2\left[\right.$ lactic acid- $\left.\mathrm{OH}^{-}\right]$form inactivates hydroxyl groups of PLLA and byproducts which are capable of backbiting reaction and hydrolysis of ester linkages incorporated into the polymer backbone [31-34]. It seemed that the $\mathrm{Mg}(\mathrm{OH})_{2}$ in PLLA90/PLCL10/Mg5 matrix inhibited consecutive ester pyrolysis and thermalhydrolysis reactions with binding anionic terminal group of polymer and acidic byproducts generated during thermal processing. However, the conclusive mechanism was not fully understood yet. The inorganic $\mathrm{Mg}(\mathrm{OH})_{2}$ particles slightly enhanced the tensile strength affected by molecular weight, but reduced the elongation at break due to poor interfacial adhesion, as shown in Fig. 6c. PLLA100/ Mg5 matrix exhibited higher tensile strength than PLLA but still brittle fracture characteristics like PLLA. In PLLA90/PLCL10/Mg5 matrix, the tensile strength and flexibility appropriately increased due to $\mathrm{Mg}(\mathrm{OH})_{2}$ and PLCL, respectively, compared with PLLA control.
$\mathrm{Mg}(\mathrm{OH})_{2}$ not only affected the molecular weight reduction and mechanical strength under thermal processing, but also alleviated reduced $\mathrm{pH}$ value and molecular weight during degradation.

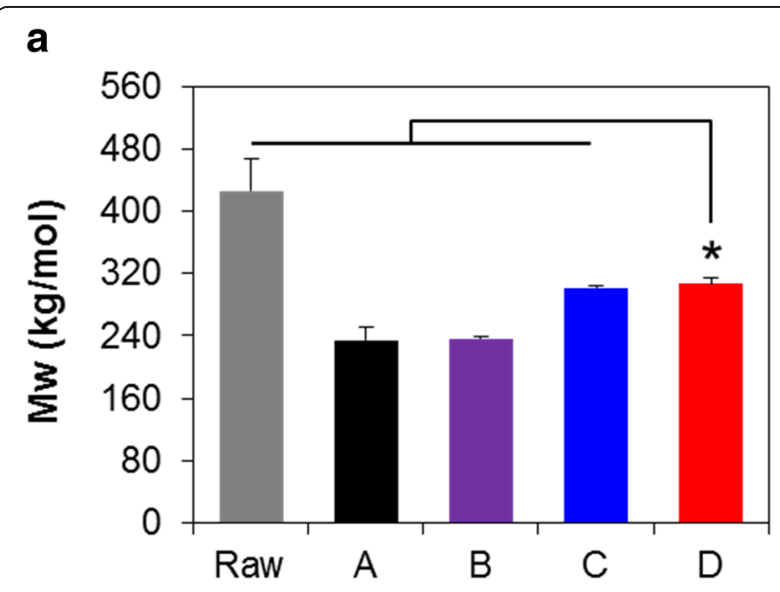

b

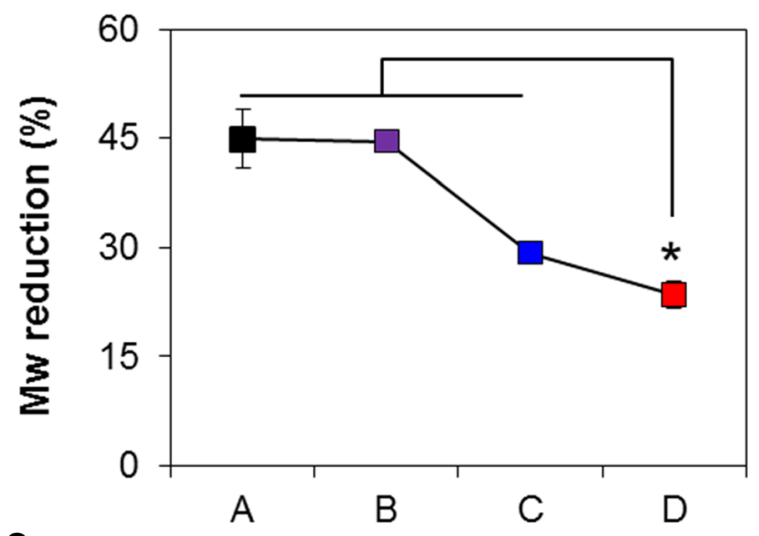

C

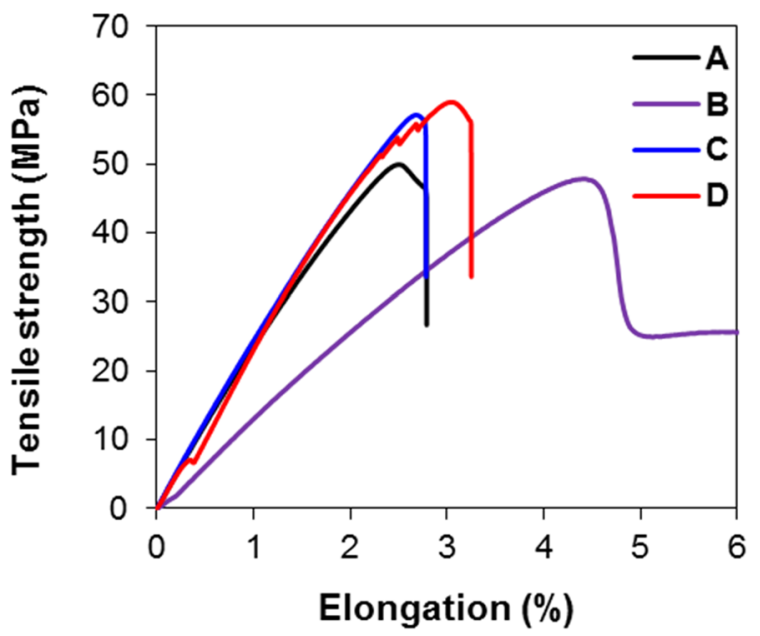

Fig. 6 Effects of the additives on PLLA based composite films on $\mathbf{a}$, b molecular weight and c tensile properties: A) processed PLLA, B) PLLA90/PLCL10, C) PLLA100/Mg5, and D) PLLA90/PLCL10/Mg5 blends $\left({ }^{*} p<0.05\right)$ 
Figure 7 displayed the results in accelerated degradation of PLLA, PLLA100/Mg5, PLLA90/PLCL10, and PLLA90/PLCL10/Mg5 blends in PBS (pH 7.4) at $60{ }^{\circ} \mathrm{C}$ for 14 days. As shown Fig. 7a, the $\mathrm{pH}$ values of media containing PLLA and PLLA90/PLCL10 samples decreased to 6.8 and 6.5, respectively because of the acidic byproducts of PLLA and PLCL. The lower $\mathrm{pH}$ value of the PLLA/PLCL degraded media depended on caprolactone segment, which was easily degenerated due to $T_{g}$ value below $60{ }^{\circ} \mathrm{C}$. Meanwhile, the $\mathrm{Mg}(\mathrm{OH})_{2}$-containing matrix such as PLLA100/Mg5 and PLLA90/PLCL10/ Mg5 slightly compensated the $\mathrm{pH}$ as compared with PLLA control, corresponding to 7.1 in 14 days. For $\mathrm{pH}$ neutralization of the degradation medium, many researchers studied some inorganic compounds such as sodium bicarbonate and calcium carbonate and incorporated these inorganic compounds into the PLLA matrix to evaluate their effect on the degradation process

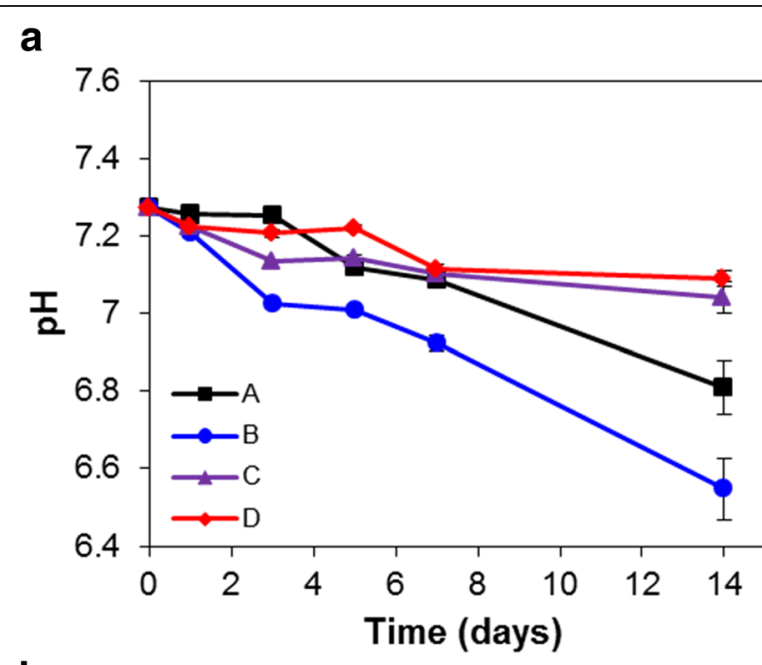

b

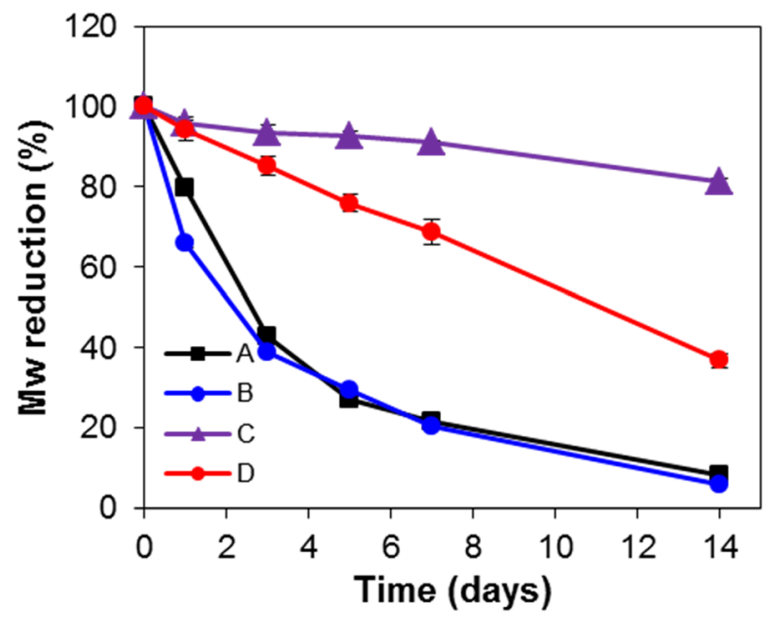

Fig. 7 Degradation study of $\mathbf{a}$ changes of $\mathrm{pH}$ and $\mathbf{b}$ reduction of molecular weight with hydrolytic time: A) processed PLLA, B) PLLA90/ PLCL10, C) PLLA100/Mg5, and D) PLLA90/PLCL10/Mg5 blends
[10, 35]. In particular, $\mathrm{Mg}(\mathrm{OH})_{2}$ particles can neutralize the acidic environment since the dehydrated magnesium ion can bind with two moles of anionic compounds [19]. The acidic degradation products of degraded PLLA and PLCL were sufficiently neutralized by $\mathrm{Mg}(\mathrm{OH})_{2}$ particles, and also the accelerated formation of degradation byproducts was prevented by binding of acidic terminal groups of PLLA and PLCL to $\mathrm{Mg}(\mathrm{OH})_{2}$.

The molecular weights of degraded samples were evaluated to confirm the effects of $\mathrm{Mg}(\mathrm{OH})_{2}$ on molecular weight reduction as well as $\mathrm{pH}$ balance (Fig. $7 \mathrm{~b}$ ). The PLLA only and PLCL90/PLCL10 blends almost degraded and their molecular weights were dropped to about $10 \%$ for 14 days. On the other hand, the molecular weight of $\mathrm{Mg}(\mathrm{OH})_{2}$-containing blends slowly declined, and, in particular, PLLA100/Mg5 scarcely decomposed as compared with other groups due to higher $T_{g}$ value of PLLA than that of PLLA90/PLCL10. During degradation, a large amount of the acidic degradation products were released, which usually resulted in a rapid decrease in the $\mathrm{pH}$ of the soaking medium [36]. The acidic byproduct reacted like a nucleophile to ester linkage of PLLA and PLCL backbones and then accelerated the decomposition of polymer chains. In PLLA blends with $\mathrm{Mg}(\mathrm{OH})_{2}$, the $\mathrm{Mg}(\mathrm{OH})_{2}$ hindered the accumulation of acidic byproducts and averted backbiting and intermolecular transesterification. These results demonstrated that the $\mathrm{Mg}(\mathrm{OH})_{2}$ affected the prevention of molecular weight reduction by neutralizing acidic substance.

\section{Conclusions}

The new PLLA blends with PLCL and $\mathrm{Mg}(\mathrm{OH})_{2}$ additives successfully fabricated by thermal processing, and the effects of PLCL and $\mathrm{Mg}(\mathrm{OH})_{2}$ additives on physicochemical and thermal properties under thermal decomposition of PLLA matrix were assessed using various analyses. With increasing the amount of PLCL, the PLLA/PLCL blends exhibited the alleviation of molecular weight reduction and the improvement of flexibility compared with thermally processed PLLA control. The PLLA/PLCL blends with increasing proportion of polycaprolactone depicted proper thermal stability as well as mechanical strength including tensile strength and elongation. In $\mathrm{Mg}(\mathrm{OH})_{2}$-containing matrix, the molecular reduction and mechanical strength were dramatically improved because the dehydrated magnesium ion inhibited the decomposition of polyester substrate by counteracting acidic compounds, which is a kind of nucleophile at ester linkage. In particular, the $\mathrm{Mg}(\mathrm{OH})_{2}$ in matrix certainly neutralized the acidic byproducts involved during polyester degradation, that caused the acid-induced inflammatory reaction in vivo. The obtained results suggested that PLCL and $\mathrm{Mg}(\mathrm{OH})_{2}$ 
additives were effective to enhance flexibility and control degradation behavior of biodegradable PLLA matrix, and therefore the PLLA/PLCL/Mg(OH $)_{2}$ composites have the potential as a material for bio-absorbable biomedical devices such as implants and stents.

\section{Competing interests}

The authors declare that they have no competing interests.

\section{Authors' contributions}

EK carried out the experiments and drafted the manuscript. EL participated in the design of the study and revised the manuscript. KH, JK and HK helped to draft and reviewed the manuscript. All authors read and approved the final manuscript.

\section{Acknowledgments}

This work was supported by Pioneer Research Center Program (2014M3C1A3056052) and the Bio \& Medical Technology Development Program (2014M3A9D3033887) through the National Research Foundation of Korea funded by the Ministry of Science, ICT \& Future Planning (MSIP) and Core Material Development Program (10048019) funded by Ministry of Trade, Industry and Energy (MOTIE), Republic of Korea.

\section{Author details}

${ }^{1}$ Center for Biomaterials, Korea Institute of Science and Technology, Hwarangno 14-gil 5, Seongbuk-gu Seoul 02792, South Korea. ${ }^{2}$ Department of Biological Science, Korea University, Anam-ro 145, Seongbuk-gu Seoul 02841, South Korea. ${ }^{3}$ Department of Biomedical Engineering, Korea University of Science and Technology, Gajeong-ro 217, Yuseong-gu Daejeon 34113, South Korea.

Received: 11 January 2016 Accepted: 26 February 2016 Published online: 15 March 2016

\section{References}

1. Ray SS, Okamoto M. Biodegradable polylactide and its nanocomposites: opening a new dimension for plastics and composites. Macromol Rapid Commun. 2003;24:815-40

2. Zhang Z, Feng S-S. The drug encapsulation efficiency, in vitro drug release, cellular uptake and cytotoxicity of paclitaxel-loaded poly (lactide)tocopheryl polyethylene glycol succinate nanoparticles. Biomaterials. 2006:27:4025-33.

3. Rasal RM, Hirt DE. Micropatterning of covalently attached biotin on poly (lactic acid) film surfaces. Macromol Biosci. 2009;9:989-96.

4. Auras R, Harte B, Selke S. An overview of polylactides as packaging materials. Macromol Biosci. 2004:4:835-64.

5. Vink ET, Rabago KR, Glassner DA, Gruber PR. Applications of life cycle assessment to NatureWorks ${ }^{\text {TM }}$ polylactide (PLA) production. Polym Degrad Stab. 2003;80:403-19.

6. $\quad Y u$ H, Huang N, Wang C, Tang Z. Modeling of poly (L-lactide) thermal degradation: Theoretical prediction of molecular weight and polydispersity index. J Appl Polym Sci. 2003;88:2557-62.

7. Jamshidi K, Hyon S-H, Ikada Y. Thermal characterization of polylactides. Polymer. 1988;29:2229-34.

8. Kopinke F-D, Remmler M, Mackenzie K, Möder M, Wachsen O. Thermal decomposition of biodegradable polyesters - II. Poly (lactic acid). Polym Degrad Stab. 1996:53:329-42

9. Amid P. Classification of biomaterials and their related complications in abdominal wall hernia surgery. Hernia. 1997;1:15-21.

10. Agrawal CM, Athanasiou KA. Technique to control pH in vicinity of biodegrading PLA-PGA implants. J Biomed Mater Res. 1997:38:105-14.

11. Linhart W, Peters F, Lehmann W, Schwarz K, Schilling AF, Amling M, et al. Biologically and chemically optimized composites of carbonated apatite and polyglycolide as bone substitution materials. J Biomed Mater Res. 2001;54:162-71.

12. Khankrua R, Pivsa-Art S, Hiroyuki H, Suttiruengwong S. Effect of chain extenders on thermal and mechanical properties of poly (lactic acid) at high processing temperatures: Potential application in PLA/Polyamide 6 blend. Polym Degrad Stab. 2014;108:232-40.
13. Lee S, Lee JW. Characterization and processing of biodegradable polymer blends of poly (lactic acid) with poly (butylene succinate adipate). Korea Aust Rheol J. 2005;17:71-7.

14. Park JW, Im SS. Phase behavior and morphology in blends of poly (L-lactic acid) and poly (butylene succinate). J Appl Polym Sci. 2002;86:647-55.

15. Li Q, Yoon J-S, Chen G-X. Thermal and biodegradable properties of poly (L-lactide)/poly ( $\varepsilon$-Caprolactone) compounded with functionalized organoclay. J Polym Environ. 2011;19:59-68.

16. Wang L, Ma W, Gross R, McCarthy S. Reactive compatibilization of biodegradable blends of poly (lactic acid) and poly ( $\varepsilon$-caprolactone). Polym Degrad Stab. 1998:59:161-8.

17. Wu D, Zhang Y, Zhang M, Yu W. Selective localization of multiwalled carbon nanotubes in poly ( $\varepsilon$-caprolactone)/polylactide blend. Biomacromolecules. 2009;10:417-24

18. Gu X-N, Li S-S, Li X-M, Fan Y-B. Magnesium based degradable biomaterials: A review. Front Mater Sci. 2014,8:200-18.

19. Kum $\mathrm{CH}$, Cho Y, Joung YK, Choi J, Park K, Seo SH, et al. Biodegradable poly (I-lactide) composites by oligolactide-grafted magnesium hydroxide for mechanical reinforcement and reduced inflammation. J Mater Chem B. 2013:1:2764-72.

20. Wen W, Luo B, Qin X, Li C, Liu M, Ding S, et al. Strengthening and toughening of poly (L-lactide) composites by surface modified MgO whiskers. Appl Surf Sci. 2015;332:215-23.

21. Kaci M, Benhamida A, Zaidi L, Touati N, Remili C. Photodegradation of Poly (Lactic Acid)/Organo-Modified Clay Nanocomposites under Natural Weathering Exposure. Ecosustainable Polymer Nanomaterials for Food Packaging: Innovative Solutions, Characterization Needs, Safety and Environmental Issues. 2013: 281

22. Scoponi M, Pradella F, Kaczmarek H, Amadelli R, Carassiti V. A reappraisal of the photo-oxidation mechanism at short and long wavelengths for poly (2, 6-dimethyl-1, 4-phenylene oxide). Polymer. 1996;37:903-16.

23. Li AD, Sun Z, Zhou M, Xu X, Ma J, Zheng W, et al. Electrospun Chitosangraft-PLGA nanofibres with significantly enhanced hydrophilicity and improved mechanical property. Colloids Surf B. 2013;102:674-81.

24. Coltelli M-B, Toncelli C, Ciardelli F, Bronco S. Compatible blends of biorelated polyesters through catalytic transesterification in the melt. Polym Degrad Stab. 2011;96:982-90.

25. Sivalingam G, Karthik R, Madras G. Kinetics of thermal degradation of poly (E-caprolactone). J Anal Appl Pyrolysis. 2003;70:631-47.

26. Douglas JF. A dynamic measure of order in structural glasses. Comput Mater Sci. 1995:4:292-308

27. Seidel A. Processing and finishing of polymeric materials Vol. 2. New Jersey, NJ: Wiley; 2011.

28. Lim L-T, Auras R, Rubino M. Processing technologies for poly (lactic acid). Prog Polym Sci. 2008;33:820-52.

29. Draye A-C, Persenaire O, Brožek J, Roda J, Košek T, Dubois $P$. Thermogravimetric analysis of poly ( $\varepsilon$-caprolactam) and poly [( $\varepsilon$-caprolactam)-co-( $\varepsilon$-caprolactone)] polymers. Polymer. 2001:42:8325-32.

30. Persenaire $O$, Alexandre $M$, Degée $P$, Dubois $P$. Mechanisms and kinetics of thermal degradation of poly ( $\varepsilon$-caprolactone). Biomacromolecules. 2001;2:288-94

31. Pampaloni F, Reynaud EG, Stelzer EH. The third dimension bridges the gap between cell culture and live tissue. Nat Rev Mol Cell Biol. 2007:8:839-45.

32. Hutmacher DW. Biomaterials offer cancer research the third dimension. Nat Mater. 2010:9:90-3.

33. DesRochers TM, Palma E, Kaplan DL. Tissue-engineered kidney disease models. Adv Drug Deliv Rev. 2014:69:67-80.

34. Garrod M, San Chau DY. An Overview of Tissue Engineering as an Alternative for Toxicity Assessment. J Pharm Pharm Sci. 2016;19:31-71.

35. Van der Meer S, De Wijn J, Wolke J. The influence of basic filler materials on the degradation of amorphous D-and L-lactide copolymer. J Mater Sci Mater Med. 1996;7:359-61.

36. Gibbons D. Tissue response to resorbable synthetic polymers. Degradation phenomena on polymeric biomaterials: Springer; 1992. p. 97-105. 